\title{
Recent Advances in Optical Limiting Properties of Fluorinated Graphene Oxides
}

Panit Chantharasupawong ${ }^{1}$, Reji Philip ${ }^{2}$, Narayanan T. Narayanan ${ }^{3}$, Parambath M. Sudeep $^{4}$, Akshay Mathkar ${ }^{3}$, Pulickel M. Ajayan ${ }^{3}$, and Jayan Thomas ${ }^{1,5,6}$

1 College of Optics and Photonics, CREOL, University of Central Florida, Orlando, FL 32816, USA

2 Raman Research Institute, C.V. Raman Avenue, Sadashivanagar, Bangalore 560080, India.

3 Department of Mechanical Engineering and Materials Science, Rice University, Houston, TX 77005, USA

4 Department of Physics, Cochin University of Science and Technology, Kochi 682 022, India. 5 NanoScience Technology Center, University of Central Florida, Orlando, FL 32826, USA 6 Department of Material Science and Engineering, University of Central Florida, Orlando, FL 32816, USA

\begin{abstract}
There is a substantial interest in finding materials with high nonlinear optical (NLO) properties of materials because of its attractive applications in optical limiting for safety protections. In an effort to develop highly performing optical limiting materials, recently we have found that fluorination of graphene oxides leads to improvement in their NLO properties.
\end{abstract}

KEYWORDS: Fluorination, Photoacoustic, Z-scan, localized sp² domains, Graphene Oxide,Opitcal limiting.

\section{INTRODUCTION}

With the aid of rapid theoretical and experimental developments in nanoscience and nanotechnology, a number of novel nanomaterials with exceptional physical and chemical properties have been synthesized. One of the well-known nanomaterials is the family of carbon allotropes. Before the advent of nanotechnology only two

Optical Processes in Organic Materials and Nanostructures II, edited by Manfred Eich, Jean-Michel Nunzi, Rachel Jakubiak, Proc. of SPIE

Vol. 8827, 88270G · C 2013 SPIE · CCC code: 0277-786X/13/\$18 · doi: 10.1117/12.2023768

Proc. of SPIE Vol. $882788270 \mathrm{G}-1$ 
allotropes of carbon were known: graphite and diamond. However, several allotropes of carbon have been subsequently found. These materials differ not only in their atomic structures but also in their physical properties.

Nanomaterials can be categorized by their dimensionality, which ranges from zero dimension (0D) to three dimension (3D). In the case of carbon the common structures are, for example, OD fullerene, 1D carbon nanotube, 2D graphene, and 3D graphite. Interestingly, one can think of 2D graphene as a building block to form the other dimensional structures of carbon allotropes. Graphene is a monolayer of carbon atoms densely packed into a twodimensional honeycomb lattice. Graphene can be wrapped up to form 0D fullerene, rolled to form 1D nanotube, and stacked to form 3D graphite ${ }^{1}$, as illustrated in Figure 1. Graphene possesses excellent electrical properties, mechanical flexibility, optical transparency, thermal conductivity and low thermal expansion coefficient ${ }^{2-6}$. There are several ways to synthesize graphene; for example, exfoliation of natural graphite ${ }^{7}$, un-zipping of carbon nanotube $^{8}$, chemical vapor deposition ${ }^{9}$, and reduction of graphene oxide $(\mathrm{GO})^{10}$. Among these processes, reduction of GO to form graphene has attracted significant attention ${ }^{11-15}$ since this method can offer cost effective, large scale production of graphene based materials .

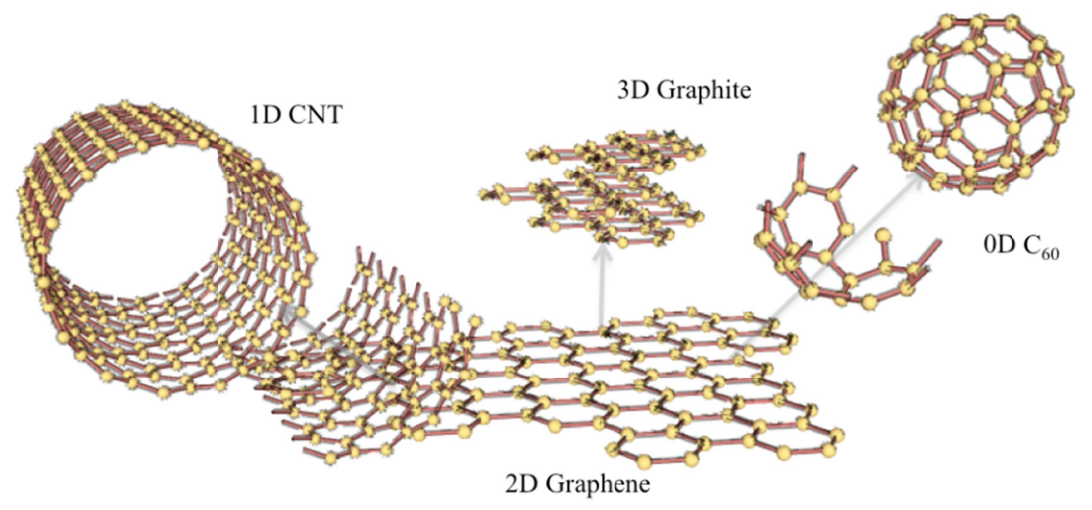

Figure1. Graphene is a basic building block of fullerene, carbon nanotube and graphite.

GO is an electrically hybrid material between the conducting $\pi$-states of $\mathrm{sp}^{2}$ carbon sites, which contribute to the bandgap formation of the material, and the $\sigma$-states of $\mathrm{sp}^{3}$ carbon sites. Chemical reduction can transform GO from an insulator to semiconductor and also to a metal-like state, in the form of graphene. Besides the excellent properties of its final product graphene, the GO precursor has interesting properties by itself. Unlike graphene, GO 
has several types of oxygen containing functional moieties such as hydroxyl, epoxide, diol, ketone and carboxyl. These functional groups allow GO to interact with a wide range of materials, both organic and inorganic, which results in the high processability of GO. Functionalization of GO, both covalently and non-covalently, also allows manipulation of GO properties. For example, cross-linking polyallylamine with GO results in improved stiffness and strength ${ }^{16}$. Grafting a polymer to GO can improve dispersibility of the material in liquid media ${ }^{17}{ }^{18}$. Chitosangrafted GO exhibits excellent biocompatibility ${ }^{19}$. Optical limiting property of GO also greatly enhances with both organic and inorganic decorations ${ }^{20-22}$. Therefore, due to its versatility, functionalized-GOs are promising candidates in various applications, for instance drug delivery ${ }^{23}{ }^{24}$, magnetic resonance imaging (MRI) ${ }^{25}$, memory devices $^{26}$, supercapacitor $^{27,28}$, and optoelectronic devices ${ }^{29,30}$.

Fluorination of carbon nanomaterials has many advantages due to the unique nature of the carbon-fluorine (C-F) bond. For instance, the C-F bond demonstrates excellent oxidative and thermal stability ${ }^{31}$. Fluorine atoms being the most electronegative element in the periodic table, C-F bonds have high polarity and low surface free energy. F-GO will be an attractive material in many applications such as super amphiphobic surfaces, multimodality imaging and photonic devices.

Optical limiting is a nonlinear optical process in which the transmittance of a material decreases as the input light intensity increases. Materials with optical limiting property can be used to fabricate laser protection devices. In this paper we investigate the effect of fluorination on the optical limiting (OL) properties of GO dispersed in water,,for nanosccond laser pulses at the excitation wavelength of $532 \mathrm{~nm}$

\section{EXPERIMENTAL SECTION}

All Graphene oxide (GO) based samples were synthesized. Sample characterization and synthesis methods can be found in our previous reports ${ }^{32,33}$. We measured optical limiting of three samples namely, graphene oxides (GO), fluorinated graphene oxide (F-GO), and highly fluorinated graphene oxides (HF-GO). While the first two are dispersed in water, HF-GO is dispersed in N-methyl-2-pyrrolidinone (NMP) due to its high polarity. The open aperture Z-scan technique ${ }^{34}$ was used to measure optical limiting properties at 532nm wavelength and 5ns laser pulse if these samples. All samples were prepared to have a linear transmission of $50 \%$ at the excitation wavelength 
of $532 \mathrm{~nm}$. A photodiode was mounted near the sample at an angle to the beam axis to monitor linear and/or nonlinear light scattering. The obtained z-scans curves for an input energy of $30 \mu \mathrm{J}$ are shown Figure 2(a). All samples show an optical limiting (OL) property since the transmission decreases with increase in input fluence.

(a)

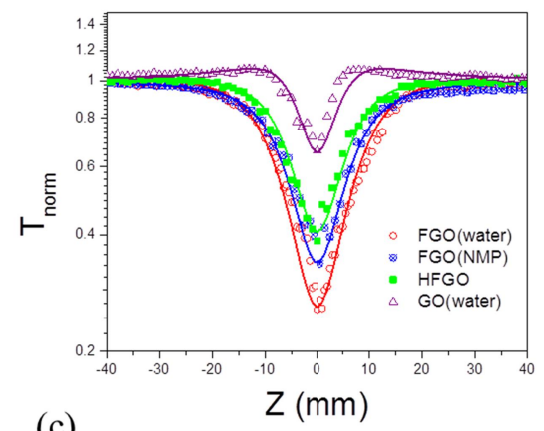

(c)

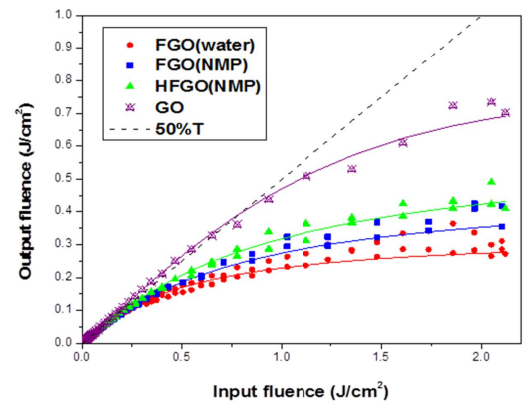

(b)

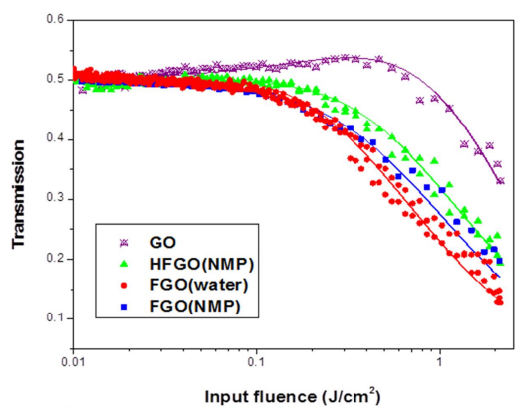

(d)

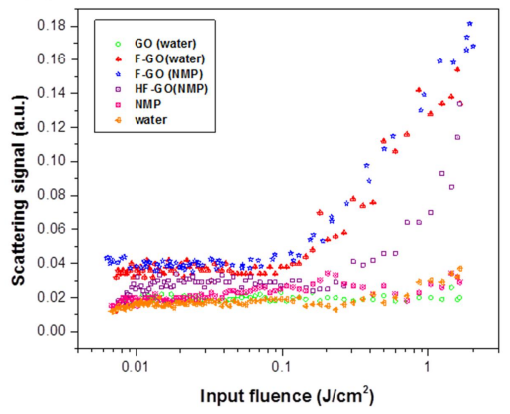

Figure2. (a) Z-scan curves of the samples. (b) Transmission vs input fluence (c) Output fluence vs input fluence (d)Scattering signals.

The z-scan data can be used to plot the variation of sample transmission with input laser fluence, because for a spatially Gaussian beam, the light fluence $F_{\text {in }}(z)$ at any position $z$ can be calculated from the corresponding beam radius $w(\mathrm{z})$ and the input laser pulse energy $E_{\text {in }}$. The plot of sample transmission versus input fluence is shown in Figure 2(b), and that of output fluence versus input fluence is shown in Figure 2(c).

It is found that optical limiting efficiencies of all fluorinated graphene samples are higher than that of GO in water. The optical limiting threshold (input fluence at which transmission decreases to $50 \%$ of the linear transmission due to the nonlinearity) of F-GO/water and $\mathrm{H}-\mathrm{FGO} / \mathrm{NMP}$ are at 0.8 and $1.5 \mathrm{~J} / \mathrm{cm}^{2}$ respectively. The optical limiting properties of F-GO in (N-methyl-2-pyrrolidinone) NMP were also studied to find out the solvent 
contribution to the optical limiting performances when comparing F-GO/water to HF-GO/NMP dispersions. It is found that F-GO in NMP also has better optical limiting efficiency than HF-GO in NMP.

Optical limiting behavior can arise from nonlinear absorption and/or nonlinear scattering. For example, carbon particle suspensions (CS) show strong optical limiting due to NLS caused by thermally induced microplasma, ${ }^{35}$ whereas fullerenes $\left(\mathrm{C}_{60}\right)$ exhibit robust limiting due to large RSA. ${ }^{36-38}$ In addition, multiwalled and single walled carbon nanotubes (CNTs) exhibit broadband optical limiting due to NLS ${ }^{39,40}$ while GO exhibits limiting due to RSA. ${ }^{41}$

By measuring the scattered light from our samples, it is found that all fluorinated graphene oxide samples have strong nonlinear scattering whereas there is no nonlinear scattering present in GO, Figure 2(d). This finding suggests that there is a significant enhancement in nonlinear scattering due to the presence of C-F bonds in the fluorinated samples. This enhancement, as a result, contributes to better optical limiting properties.

However, when comparing the z-scan and scattering data, it is found that, for fluorinated graphene oxide samples, the onsets of the optical limiting start earlier than the onsets of nonlinear scattering. This observation suggests that the optical limiting action of fluorinated graphene oxide in the $n s$ excitation regime is not exclusively due to nonlinear scattering. In fact, Photoacoustic measurement technique is employed to verify this assumption.

Photoacoustics (PA) is a well-known sensitive method for measuring low level absorptions in materials. ${ }^{42-46}$ In our PA measurements, an in-house fabricated PA cell was used. The detail of the setup can be found elsewhere ${ }^{33}$. The normalized PA signal plotted against input energy is shown in Figure 3. According to the plot, the PA signal is the highest in F-GO while GO has the lowest PA signal and HF-GO assumes an intermediate value. This observation renders the fact that, besides NLS, NLA also plays a significant role in the optical limiting of fluorinated GO samples.

Proc. of SPIE Vol. $882788270 \mathrm{G}-5$ 


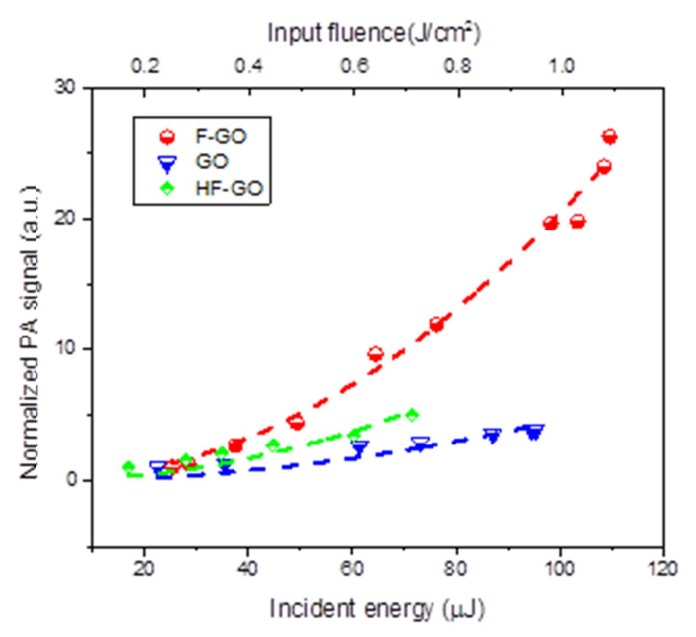

Figure3. Normalized photo acoustic signals

X. F. Jiang et al. reported significantly enhanced nonlinear absorption of GO upon partial reduction. ${ }^{42}$ This enhancement in nonlinear absorption was attributed to localized $\mathrm{sp}^{2}$ domains. By studying X-ray Photoelectron Spectroscopy (XPS) data of these samples ${ }^{33}$, it is found that the degrees of the $\mathrm{sp}^{2}$ localizations in our GO and F-GO samples are similar, while HF-GO has lowest number of $\mathrm{sp}^{2}$ to $\mathrm{sp}^{3}$ carbons. This renders the significance of fluorine $\mathrm{sp}^{3}$ sites in the enhancement of nonlinear optical properties of fluorinated samples.

\section{CONCLUSIONS}

In conclusion, it is found that the optical limiting efficiency of F-GO was significantly greater than of GO. Nonlinear scattering measurements reveal that scattering is higher in F-GO when compared to GO, while photoacoustic measurements indicate that nonlinear absorption is also superior in F-GO. The limiting threshold of F$\mathrm{GO}$ is an order of magnitude higher than of GO. These observations suggest that F-GO dispersions are potential candidates for optical limiting applications.

\section{REFERENCES}

[1] Geim, A.K., and Novoselov, K.S., "The rise of graphene", Nat Mater 6 (3), 183-191 (2007)

[2] Castro Neto, A.H., Guinea, F., Peres, N.M.R., Novoselov, K.S., and Geim, A.K., "The electronic properties of graphene", Reviews of Modern Physics 81 (1), 109-162 (2009)

[3] Chen, H., Müller, M.B., Gilmore, K.J., Wallace, G.G., and Li, D., "Mechanically Strong, Electrically Conductive, and Biocompatible Graphene Paper", Advanced Materials 20 (18), 3557-3561 (2008)

[4] Li, X., Zhang, G., Bai, X., Sun, X., Wang, X., Wang, E., and Dai, H., "Highly conducting graphene sheets and Langmuir-Blodgett films", Nat Nano 3 (9), 538-542 (2008) 
[5] Bae, S., Kim, H., Lee, Y., Xu, X., Park, J.-S., Zheng, Y., Balakrishnan, J., Lei, T., Ri Kim, H., Song, Y.I., Kim, Y.-J., Kim, K.S., Ozyilmaz, B., Ahn, J.-H., Hong, B.H., and Iijima, S., "Roll-to-roll production of 30-inch graphene films for transparent electrodes", Nat Nano 5 (8), 574-578 (2010)

[6] Balandin, A.A., Ghosh, S., Bao, W., Calizo, I., Teweldebrhan, D., Miao, F., and Lau, C.N., "Superior Thermal Conductivity of Single-Layer Graphene", Nano Letters 8 (3), 902-907 (2008)

[7] Hernandez, Y., Nicolosi, V., Lotya, M., Blighe, F.M., Sun, Z., De, S., McGovern, I.T., Holland, B., Byrne, M., Gun'Ko, Y.K., Boland, J.J., Niraj, P., Duesberg, G., Krishnamurthy, S., Goodhue, R., Hutchison, J., Scardaci, V., Ferrari, A.C., and Coleman, J.N., "High-yield production of graphene by liquid-phase exfoliation of graphite", Nat Nano 3 (9), 563-568 (2008)

[8] Kosynkin, D.V., Higginbotham, A.L., Sinitskii, A., Lomeda, J.R., Dimiev, A., Price, B.K., and Tour, J.M., "Longitudinal unzipping of carbon nanotubes to form graphene nanoribbons", Nature 458 (7240), 872-876 (2009)

[9] Reina, A., Jia, X., Ho, J., Nezich, D., Son, H., Bulovic, V., Dresselhaus, M.S., and Kong, J., "Large Area, Few-Layer Graphene Films on Arbitrary Substrates by Chemical Vapor Deposition", Nano Letters 9 (1), 30-35 (2008)

[10] Stankovich, S., Dikin, D.A., Piner, R.D., Kohlhaas, K.A., Kleinhammes, A., Jia, Y., Wu, Y., Nguyen, S.T., and Ruoff, R.S., "Synthesis of graphene-based nanosheets via chemical reduction of exfoliated graphite oxide", Carbon 45 (7), 1558-1565 (2007)

[11] Chen, W., Yan, L., and Bangal, P.R., "Preparation of graphene by the rapid and mild thermal reduction of graphene oxide induced by microwaves", Carbon 48 (4), 1146-1152 (2010)

[12] Eda, G., Fanchini, G., and Chhowalla, M., "Large-area ultrathin films of reduced graphene oxide as a transparent and flexible electronic material", Nat Nano 3 (5), 270-274 (2008)

[13] Eda, G., Lin, Y.-Y., Miller, S., Chen, C.-W., Su, W.-F., and Chhowalla, M., "Transparent and conducting electrodes for organic electronics from reduced graphene oxide", Applied Physics Letters 92 (23), 233305-233303 (2008)

[14] Gao, W., Alemany, L.B., Ci, L., and Ajayan, P.M., "New insights into the structure and reduction of graphite oxide", Nat Chem 1 (5), 403-408 (2009)

[15] Xu, C., Wang, X., and Zhu, J., "Graphene-Metal Particle Nanocomposites", The Journal of Physical Chemistry C 112 (50), 19841-19845 (2008)

[16] Park, S., Dikin, D.A., Nguyen, S.T., and Ruoff, R.S., "Graphene Oxide Sheets Chemically Cross-Linked by Polyallylamine", The Journal of Physical Chemistry C 113 (36), 15801-15804 (2009)

[17] Qi, X., Pu, K.-Y., Zhou, X., Li, H., Liu, B., Boey, F., Huang, W., and Zhang, H., "Conjugated-

Polyelectrolyte-Functionalized Reduced Graphene Oxide with Excellent Solubility and Stability in Polar Solvents",

Small 6 (5), 663-669 (2010)

[18] Stankovich, S., Piner, R.D., Nguyen, S.T., and Ruoff, R.S., "Synthesis and exfoliation of isocyanate-treated graphene oxide nanoplatelets", Carbon 44 (15), 3342-3347 (2006)

[19] Rana, V.K., Choi, M.-C., Kong, J.-Y., Kim, G.Y., Kim, M.J., Kim, S.-H., Mishra, S., Singh, R.P., and Ha, C.-S., "Synthesis and Drug-Delivery Behavior of Chitosan-Functionalized Graphene Oxide Hybrid Nanosheets", Macromolecular Materials and Engineering 296 (2), 131-140 (2011)

[20] Liu, Y., Zhou, J., Zhang, X., Liu, Z., Wan, X., Tian, J., Wang, T., and Chen, Y., "Synthesis, characterization and optical limiting property of covalently oligothiophene-functionalized graphene material", Carbon 47 (13), 3113-3121 (2009)

[21] Xu, Y., Liu, Z., Zhang, X., Wang, Y., Tian, J., Huang, Y., Ma, Y., Zhang, X., and Chen, Y., "A Graphene Hybrid Material Covalently Functionalized with Porphyrin: Synthesis and Optical Limiting Property", Advanced Materials 21 (12), 1275-1279 (2009)

[22] Balapanuru, J., Yang, J.-X., Xiao, S., Bao, Q., Jahan, M., Polavarapu, L., Wei, J., Xu, Q.-H., and Loh, K.P., "A Graphene Oxide-Organic Dye Ionic Complex with DNA-Sensing and Optical-Limiting Properties", Angewandte Chemie 122 (37), 6699-6703 (2010)

[23] Sun, X., Liu, Z., Welsher, K., Robinson, J., Goodwin, A., Zaric, S., and Dai, H., "Nano-graphene oxide for cellular imaging and drug delivery", Nano Research 1 (3), 203-212 (2008)

[24] Yang, X., Zhang, X., Ma, Y., Huang, Y., Wang, Y., and Chen, Y., "Superparamagnetic graphene oxideFe3O4 nanoparticles hybrid for controlled targeted drug carriers", Journal of Materials Chemistry 19 (18), 2710 2714 (2009) 
[25] Ma, X., Tao, H., Yang, K., Feng, L., Cheng, L., Shi, X., Li, Y., Guo, L., and Liu, Z., "A functionalized graphene oxide-iron oxide nanocomposite for magnetically targeted drug delivery, photothermal therapy, and magnetic resonance imaging", Nano Research 5 (3), 199-212 (2012)

[26] Zhuang, X.-D., Chen, Y., Liu, G., Li, P.-P., Zhu, C.-X., Kang, E.-T., Noeh, K.-G., Zhang, B., Zhu, J.-H., and Li, Y.-X., "Conjugated-Polymer-Functionalized Graphene Oxide: Synthesis and Nonvolatile Rewritable Memory Effect", Advanced Materials 22 (15), 1731-1735 (2010)

[27] Wang, H., Hao, Q., Yang, X., Lu, L., and Wang, X., "Graphene oxide doped polyaniline for supercapacitors", Electrochemistry Communications 11 (6), 1158-1161 (2009)

[28] Chen, S., Zhu, J., Wu, X., Han, Q., and Wang, X., "Graphene Oxide-MnO2 Nanocomposites for Supercapacitors", ACS Nano 4 (5), 2822-2830 (2010)

[29] Liu, Z., Liu, Q., Huang, Y., Ma, Y., Yin, S., Zhang, X., Sun, W., and Chen, Y., "Organic Photovoltaic Devices Based on a Novel Acceptor Material: Graphene", Advanced Materials 20 (20), 3924-3930 (2008)

[30] Yu, D., Yang, Y., Durstock, M., Baek, J.-B., and Dai, L., "Soluble P3HT-Grafted Graphene for Efficient

Bilayer-Heterojunction Photovoltaic Devices", ACS Nano 4 (10), 5633-5640 (2010)

[31] Johns, I.B., McElhill, E.A., and Smith, J.O., "Thermal Stability of Some Organic Compounds", Journal of Chemical \& Engineering Data 7 (2), 277-281 (1962)

[32] Mathkar, A., Narayanan, T.N., Alemany, L.B., Cox, P., Nguyen, P., Gao, G., Chang, P., Romero-Aburto, R., Mani, S.A., and Ajayan, P.M., "Synthesis of Fluorinated Graphene Oxide and its Amphiphobic Properties", Particle \& Particle Systems Characterization 30 (3), 266-272 (2013)

[33] Chantharasupawong, P., Philip, R., Narayanan, N.T., Sudeep, P.M., Mathkar, A., Ajayan, P.M., and Thomas, J., "Optical Power Limiting in Fluorinated Graphene Oxide: An Insight into the Nonlinear Optical Properties", The Journal of Physical Chemistry C 116 (49), 25955-25961 (2012)

[34] Sheik-Bahae, M., Said, A.A., Wei, T.H., Hagan, D.J., and Van Stryland, E.W., "Sensitive measurement of optical nonlinearities using a single beam", Quantum Electronics, IEEE Journal of 26 (4), 760-769 (1990)

[35] Mansour, K., Soileau, M.J., and Stryland, E.W.V., "Nonlinear optical properties of carbon-black suspensions (ink)", J. Opt. Soc. Am. B 9 (7), 1100-1109 (1992)

[36] Justus, B.L., Kafafi, Z.H., and Huston, A.L., "Excited-state absorption-enhanced thermal optical limiting in C60", Opt. Lett. 18 (19), 1603-1605 (1993)

[37] Golovlev, V.V., Garrett, W.R., and Chen, C.H., "Reverse saturable absorption of C60 in liquids irradiated by picosecond and nanosecond laser pulses", J. Opt. Soc. Am. B 13 (12), 2801-2806 (1996)

[38] Sun, Y.-P., Riggs, J.E., and Liu, B., "Optical Limiting Properties of [60]Fullerene Derivatives", Chemistry of Materials 9 (5), 1268-1272 (1997)

[39] Vivien, L., Anglaret, E., Riehl, D., Bacou, F., Journet, C., Goze, C., Andrieux, M., Brunet, M., Lafonta, F., Bernier, P., and Hache, F., "Single-wall carbon nanotubes for optical limiting", Chemical Physics Letters 307 (5-6), 317-319 (1999)

[40] Sun, X., Yu, R.Q., Xu, G.Q., Hor, T.S.A., and Ji, W., "Broadband optical limiting with multiwalled carbon nanotubes", Applied Physics Letters 73 (25), 3632-3634 (1998)

[41] Liu, Z., Wang, Y., Zhang, X., Xu, Y., Chen, Y., and Tian, J., "Nonlinear optical properties of graphene oxide in nanosecond and picosecond regimes", Applied Physics Letters 94 (2), 021902-021903 (2009)

[42] Alan McDonald, F., "Photoacoustic determination of small optical absorption coefficients: extended theory", Appl. Opt. 18 (9), 1363-1367 (1979)

[43] Hordvik, A., and Schlossberg, H., "Photoacoustic technique for determining optical absorption coefficients in solids", Appl. Opt. 16 (1), 101-107 (1977)

[44] Rosencwaig, A., and Hindley, T.W., "Photoacoustic measurement of low-level absorptions in solids", Appl. Opt. 20 (4), 606-609 (1981)

[45] Tam, A.C., "Applications of photoacoustic sensing techniques", Reviews of Modern Physics 58 (2), 381 431 (1986)

[46] Sigrist, M.W., and Kneubuhl, F.K., "Laser-generated stress waves in liquids", J. Acoust Soc. Am. 64 (6), 1652-1978 (Dec. 1978) 